\title{
Optical Follow up Photometry of the Transiting Extrasolar Planet XO-2
}

\author{
Jose M. Fernandez ${ }^{1}$, Matthew J. Holman ${ }^{1}$ and Joshua N. Winn ${ }^{2}$ \\ ${ }^{1}$ Harvard-Smithsonian Center for Astrophysics, \\ 60 Garden St., Cambridge, MA 02138, USA \\ email: jfernand, mholman@cfa.harvard.edu \\ ${ }^{2}$ Department of Physics, Massachusetts Institute of Technology, \\ Cambridge, MA 02139, USA \\ email: jwinn@space.mit.edu
}

\begin{abstract}
We present three full z-band transit light curves for the extrasolar planet XO-2 obtained with KeplerCam and the FLWO 1.2m telescope. The system parameters were determined fitting the data to transit models using a Markov Chain Monte Carlo simulation (MCMC). The main results presented in this poster are revised values for the parameters $R_{p} / R_{s}, a / R_{s}$ and $b$.
\end{abstract}

\section{Overview}

We present preliminary results of an ongoing TLC (Holman et al. 2006) campaign to obtain high quality transit photometry of the extrasolar planet XO-2b (Burke et al. 2007). The main goal is to measure precise transit times and, if possible, refine the system physical parameters. In this work we describe the analysis of three high $\mathrm{S} / \mathrm{N}$ transit light curves. The transits were observed in the $\mathrm{z}$ band with KeplerCam and the FLWO $1.2 \mathrm{~m}$ telescope. By combining high cadence light curves is possible to average the random noise and reduce the scatter significantly (Fig. 1 and 2)

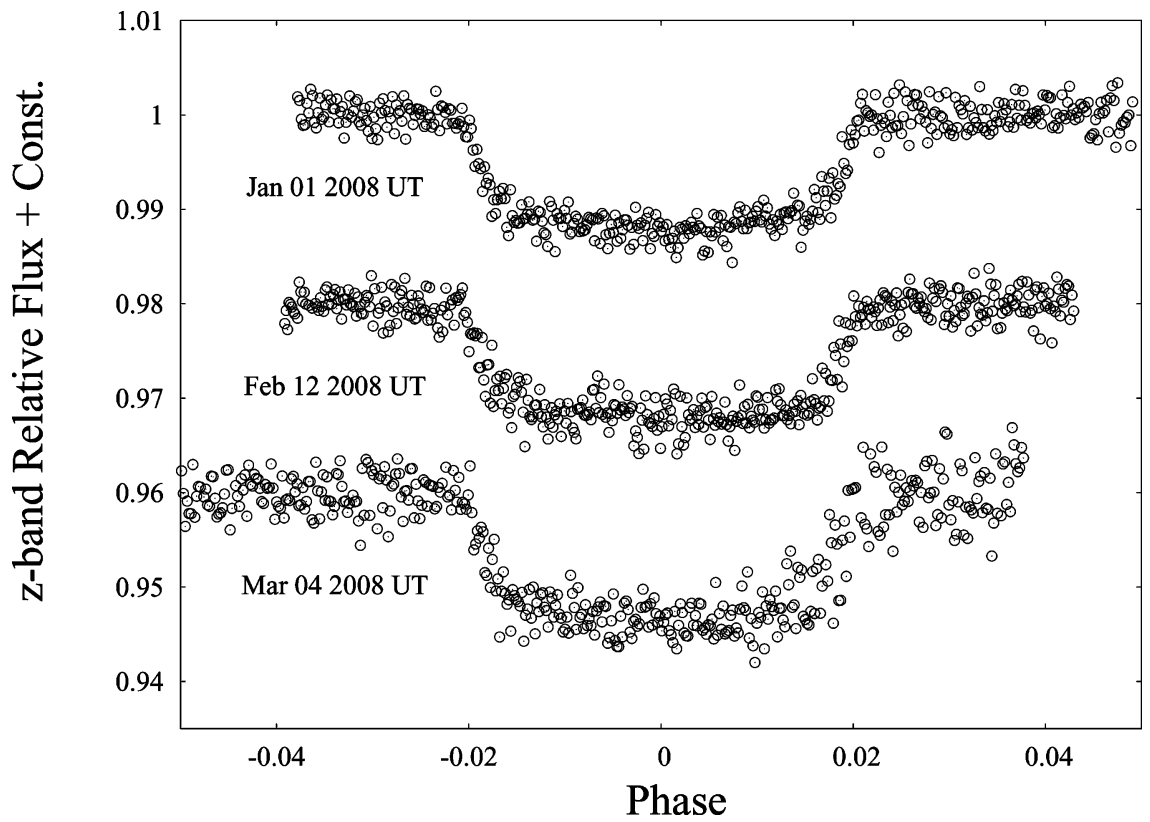

Figure 1. Individual light curves 


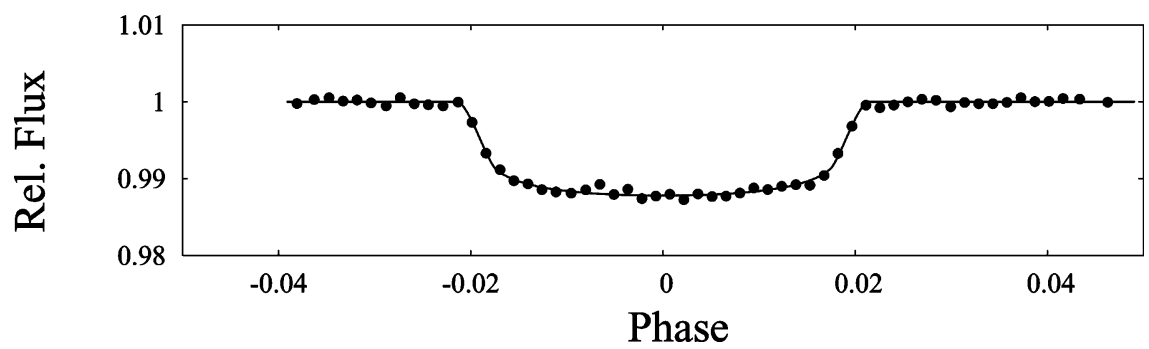

Figure 2. Combined light curves, 16x binning
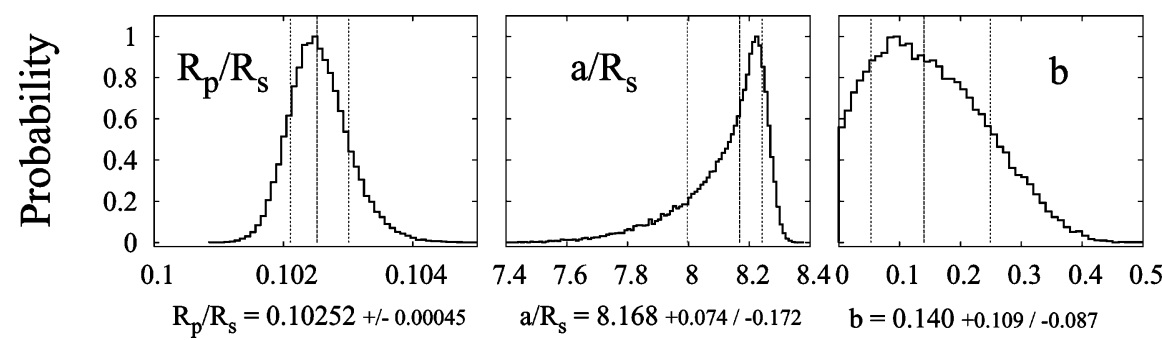

Figure 3. Light curve MCMC modeling results

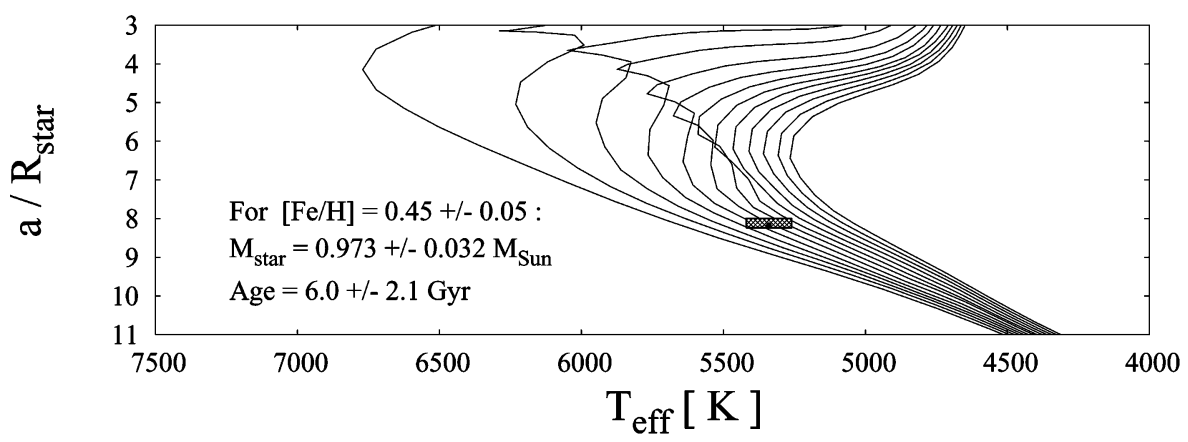

Figure 4. $\mathrm{Y}^{2}$ isochrone fit

The data was analyzed using the transit models of Mandel \& Agol (2002) and a Markov-chain Monte Carlo simulation (MCMC) to obtain the radius ratio between the planet and the star $R_{p} / R_{s}$, the ratio between the orbit's semi major axis and the radius of the star $a / R_{s}$, and the impact parameter $b$ (Fig. 3). The mass $\left(M_{s}\right)$ and age of the primary star were estimated by comparing its density (related to $a / R_{s}$ ), metallicty $([\mathrm{Fe} / \mathrm{H}]=0.45 \pm 0.05)$ and temperature $($ Teff $=5340+/-80 \mathrm{~K})$ (Burke et al. 2007, Torres et al. 2008) with the values predicted by stellar models, in this case the $\mathrm{Y}^{2}$ isochrones (Demarque et al. 2004). (Fig. 4)

Once $M_{s}$ was known, the planet's mass $M_{p}$ and the semi major axis a could be derived using the orbit's period $(P=2.61587 d)$ and it's radial velocity semi amplitude $\left(K_{s}=\right.$ $85 \pm 8 \mathrm{~m} \mathrm{~s}^{-1}$ ) (Burke et al. 2007) together with Newton and Kepler's laws. Finally, the radii of the star and planet were obtained combining $a, a / R_{s}$ and $R_{p} / R_{s}$. (Fig. 5) 

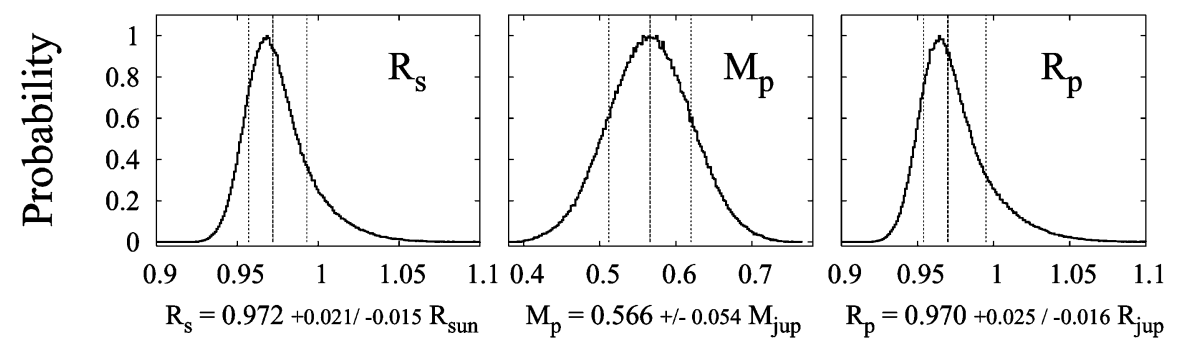

Figure 5. Mass and Radius

\section{References}

Burke, C., McCullough, P., Valenti, J., Johns-Krull, C., Janes, K., Heasley, J., Summers, F., Stys, J., Bissinger, R., Fleenor, M., Foote, C., Garcia-Melendo, E., Gary, B., Howell, P., Mallia, G. Masi, G., Taylor, B., \& Vanmunster, T. 2007, ApJ, 671, 2115

Demarque, P., Woo, J., Kim, Y., \& Yi, S. 2004, ApJS, 155, 667

Holman, M., Winn, J., Latham, D., O’Donovan, F., Charbonneau, D., Bakos, G., Esquerdo, G., Hergenrother, C., Everett, M., \& Pal, A. 2006, ApJ (Letters), 653, L69

Mandel, K. \& Agol, E. 2002, ApJ (Letters), 580, L171

Torres, G., Holman, M., \& Winn, J. 2008, ApJ, 677, 1324 